\title{
Philosophiques
}

\section{Les études kierkegaardiennes au Canada}

\section{Alastair McKinnon}

Volume 9, numéro 1, avril 1982

URI : https://id.erudit.org/iderudit/203187ar

DOI : https://doi.org/10.7202/203187ar

Aller au sommaire du numéro

Éditeur(s)

Société de philosophie du Québec

ISSN

0316-2923 (imprimé)

1492-1391 (numérique)

Découvrir la revue

Citer ce document

McKinnon, A. (1982). Les études kierkegaardiennes au Canada. Philosophiques, 9(1), 147-161. https://doi.org/10.7202/203187ar d'utilisation que vous pouvez consulter en ligne.

https://apropos.erudit.org/fr/usagers/politique-dutilisation/ 


\title{
BULLETIN
}

\section{LES ÉTUDES KIERKEGAARDIENNES AU CANADA}

\author{
par Alastair McKinnon
}

Comme il arrive dans tant d'autres secteurs de l'activité canadienne, anglophones et francophones ont entrepris et poursuivi des études kierkegaardiennes indépendamment les uns des autres; aussi nous semble-t-il plus approprié de décrire ces études séparément, même si finalement nous découvrons qu'il y a une convergence de plus en plus grande des études en question quant à l'accent mis sur ces recherches et à l'intérêt qu'on y accorde, ce qui est un signe de plus de notre maturité en tant que nation.

À ce stade-ci de ma recherche, je constate que la première publication canadienne-française consacrée à Kierkegaard est l'article du père Jean Langlois, "Essai sur Crainte et Tremblement ", paru dans Sciences Ecclésiastiques en 1954. Cet article a été suivi d'une monographie par Bruno Drolet intitulée Le démoniaque chez $S$. Kierkegaard et publiée à Joliette en 1971, et de l'article de Jacques Caron, "Dialectique de la communication chez Kierkegaard ", publié dans Philosopbiques en 1976. Le titre de chacune de ces études indique clairement le sujet de celles-ci, et il ne me reste plus qu'à mentionner la contribution d'Emmanuel Trépanier, dont la préface à la monographie de Drolet peut être considérée comme un premier appui aux études kierkegaardiennes de la part d'un membre éminent et respecté de la collectivité et dont les recherches, à certains égards, sont comparables à celles que l'on trouve en milieu anglophone.

Aujourd'hui, il nous faut au moins noter que l'étude la plus significative publiée en français est celle de Maurice 
Carignan, Individu et Société chez Kierkegaard (Halifax, 1977). Cet ouvrage est remarquable à plusieurs titres. C'est la première publication, en français ou en anglais, à traiter sérieusement et en profondeur de la pensée sociale et politique de Kierkegaard. L'étude de Carignan s'inspire largement de sources danoises qui ne sont pas encore traduites, notamment En literair Anmeldelse ( Une recension littéraire», et de passages significatifs de Papirer. Carignan réfute la représentation que l'on se fait traditionnellement et trop facilement de Kierkegaard, celle d'un penseur anti-social qui ne se préoccupe nullement des dimensions proprement sociales de l'existence humaine, et il démontre que les idées de Kierkegaard en ce domaine méritent certainement d'être sérieusernent prises en considération par nos contemporains.

Par ailleurs, Carignan a récemment écrit trois autres articles. "L'Éternel comme tiers synthétisant chez Kierkegaard » a paru dans le numéro d'avril 1981 de Philosophiques et «L'ambivalence de la réflexion selon Kierkegaard » dans le numéro de mai 1981 de Église et Théologie. Le troisième article, "La communication de la vérité selon Kierkegaard ", paraîtra dans un prochain numéro de la Revue de l'Université d'Ottawa. Toutes ces études traitent de thèmes absolument fondamentaux de la pensée kierkegaardienne et devraient largement contribuer à fournir une base solide à l'approfondissement et à la compréhension de cette pensée, tant à l'intérieur qu'à l'extérieur de la collectivité canadienne-française.

Le premier Canadien anglais à s'être sérieusement intéressé à Kierkegaard semble avoir été Clare Oke, pasteur de l'Église unie du sud de l'Ontario, qui, en 1938, adressa à Swenson des questions dont quelques-unes concernant «Les Miettes philosophiques" sont examinées dans l'ouvrage de Swenson, Something about Kierkegaard (Minneapolis, 1941). Oke était un chercheur consciencieux qui s'intéressait à l'influence de Kierkegaard sur la théologie moderne, mais il était aussi plutôt réticent, et l'on est presque certain qu'il ne publia aucun de ses travaux dans ce domaine.

La première thèse de doctorat sur Kierkegaard rédigée au Canada semble avoir été celle de Donald V. Wade qui était 
alors et est toujours à l'Université de Toronto. La thèse était intitulée The Concept of Individuality in Søren Kierkegaard et fut rédigée au début des années quarante. Wade a également publié plusieurs études sur Kierkegaard au début des années cinquante et il a récemment entrepris une étude sur $O u$ bien... Ou bien. De plus, il ne faudrait pas oublier que Wade fut responsable de l'acquisition, par le Knox College de l'Université de Toronto, d'une douzaine d'exemplaires rares de la première édition de certaines œuvres de Kierkegaard.

Le premier article sur Kierkegaard publié par un Canadien fut celui de Henry Reid MacCallum, également de l'Université de Toronto. Cet article, "Kierkegaard and Levels of Existence ", qui reflète l'intérêt profond de MacCallum pour l'esthétique, est une étude sentie et pénétrante de cet aspect capital de la pensée kierkegaardienne.

Comme l'enseignement officiel de la philosophie, chez les anglophones, au cours des années quarante et cinquante, peut difficilement être accusé de manifester un préjugé favorable à Kierkegaard (j'ai encore mes notes d'un cours de janvier 1947 qui me rappellent que l'on ne pouvait écrire au tableau noir, sans faire de faute d'orthographe, les noms de Kierkegaard et de Wittgenstein), il est peut-être important de noter que G. S. Brett appuya le travail de Wade ; Brett et MacCallum furent sûrement les figures les plus marquantes en philosophie, comme le furent, en théologie, John Line et David Hay. À l'université McGill, à peu près à la même époque, R. D. MacLennan, directeur du département de Philosophie, et James S. Thomson, doyen de la faculté de Théologie, consacrèrent également des travaux à Kierkegaard et encouragèrent d'autres chercheurs à en faire autant. Il ne fait donc aucun doute qu'un appui réel a été accordé par les personnalités les plus marquantes aux chercheurs qui souhaitaient œuvrer dans ce domaine. À ce propos, nous pouvons également souligner que vers les années 1960, John A. Irving m'invita à écrire un texte sur Kierkegaard dans le cadre d'une série diffusée par la C.B.C., Architects of Modern Thought. Il est peut-être encore plus important de rappeler que, du milieu à la fin des années quarante, Lawrence Lynch du St. Michael's College présenta un cours sur l'existentialisme français contem- 
porain qui manifestait beaucoup d'attention et de sympathie à l'œuvre de Kierkegaard, ce qui amena un certain nombre d'étudiants à s'intéresser à celle-ci.

En 1963, George Price de l'Université de l'Alberta publia une introduction à l'œuvre de Kierkegaard, intitulée The Narrow Pass (Londres, 1963). Ce livre, premier ouvrage sur Kierkegaard à être publié par un universitaire travaillant au Canada, révèle une certaine profondeur dans la compréhension de l'œuvre, mais il a été critiqué pour avoir négligé l'accent mis par Kierkegaard sur le sens de l'existence et pour avoir, par la même occasion, omis le caractère pseudonymique de bon nombre de ses écrits. (Voir, plus loin, Johnson)

Entre 1963 et 1969, Kenneth Hamilton de l'Université de Winnipeg publia plusieurs articles sur la notion de péché dans l'œuvre de Kierkegaard, ainsi qu'un petit ouvrage intitulé The Promise of Kierkegaard (Philadelphie, 1969). Hamilton s'intéresse surtout à l'analyse kierkegaardienne du christianisme et à l'influence de celle-ci sur la théologie moderne et sur l'existentialisme du vingtième siècle.

Ralph H. Johnson de l'Université de Windsor a publié une étude détaillée sur l'" existence ", intitulée The Concept of Existence in the Concluding Unscientific Postscript (La Haye, 1972). Il a depuis étudié la nature et le statut des sphères de l'existence, ainsi que les notions de subjectivité et de vérité, et les relations entre celles-ci.

Harry A. Nielson, également de l'Université de Windsor, a publié un article sur l'un des principaux arguments des Miettes philosophiques; il y a quelque temps, ill a terminé un commentaire minutieux et détaillé sur cette ouvre, intitulé Where the Passion Is. Sa recherche porte principalement sur l'éthique de Kierkegaard et sur quelques-uns des nombreux points de recoupement entre la pensée de Kierkegaard et celle de Wittgenstein.

Jeremy Walker de l'université McGill a publié des études sur les notions kierkegaardiennes de paradoxe, de vérité et notamment d'éthique ; dans ce dernier cas, il interprète la notion en se référant à la fois à l'époque où a écrit Kierkegaard et à la nôtre. Il a publié un certain nombre d'articles et une 
étude sur La pureté du coeur (qui est en fait une partie de Opbyggelige Taler i forskjellig Aand), intitulée To Will One Thing (Montréal, 1972). Walker examine actuellement d'autres études abordant les thèmes en question et d'autres sujets, plus particulièrement la notion de communauté, de manière plus approfondie et plus détaillée, et nous pouvons nous attendre à ce que d'autres travaux importants de cet auteur paraissent bientôt.

Alastair McKinnon, également de l'université McGill, a rédigé sa thèse sur Kierkegaard à l'Université d'Édimbourg, en 1950 ; depuis le début des années soixante, il a participé à divers projets touchant les études kierkegaardiennes. Il a publié un certain nombre d'articles traitant surtout du soi-disant « irrationalisme » de Kierkegaard, du « statut » des œuvres signées d'un pseudonyme, du rapport de Kierkegaard à Barth, de sa conception de la philosophie, de l'évolution de sa pensée religieuse, etc. Il a créé la version assimilable par une machine des Samlede Verker de Kierkegaard qui lui a permis de faire une étude du statut relatif des pseudonymes de Kierkegaard; il a publié The Kierkegaard Indices (Leiden, 1970-75) et récemment il a utilisé certaines techniques de mise à l'échelle statistiques et multidimensionnelles afin de tenter d'obtenir une meilleure compréhension de certains concepts fondamentaux de Kierkegaard et, sur un plan plus général, de la pensée et des ouvres de celui-ci. Dernièrement, il a traduit Søren Kierkegaard's Psychology de Ib Ostenfeld (Waterloo, 1979), premier volume de The Kierkegaard Monograph Series dont il dirige la publication aux Presses de l'université Wilfrid-Laurier, le second étant la traduction par Hong et Hong de The Controversial Kierkegaard de Gregor Malantschuk (Waterloo, 1980) ; ce volume contient également une traduction d'un cours important de Malantschuk donné à l'Université de Londres et intitulé «Political and Social Aspects of Kierkegaard's Thought ». McKinnon s'occupe aussi de la publication sur microfiche des premières éditions des œuvres de Kierkegaard faisant partie de la Collection Kierkegaard-Malantschuk récemment acquise par McGill et, si les lecteurs du Conseil le lui permettent, d'une édition conventionnelle, imprimée par ordinateur, des actes de la conférence 
sur Kierkegaard tenue dernièrement à McGill. Nous reviendrons sur ces deux séries de travaux. Tout récemment, il a annoté et fait publier une nouvelle édition de Guds Uforanderlighed de Kierkegaard (Copenhague et Montréal, 1981) qui, bien qu'elle soit imprimée par ordinateur en caractères romains modernes, rétablit toutes les conventions typographiques de Kierkegaard, comprenant l'utilisation de caractères gras et de caractères "spærret".

Au moins deux thèses de doctorat ont été rédigées au Canada au cours des dix dernières années. Ces thèses sont les suivantes: Spren Kierkegaard's Conception of Temporality, par Wayne Hamilton (McGill, 1971) et The Treatment of the Theme of Suffering in Kierkegaard's Works, par Abrahim H. Khan (McGill, 1973).

Depuis qu'il a terminé sa thèse, $M$. Khan a également écrit sur la conception du mal chez Kierkegaard et sur la relation entre Religiosités A et B. Il s'intéresse aussi aux similitudes qui existent entre Kierkegaard et la pensée orientale. Tout récemment il a utilisé quelques-unes des techniques statistiques de McKinnon afin d'explorer et de montrer la structure sous-jacente au concept kierkegaardien de Salighed (béatitude).

Nous devons aussi mentionner le travail de trois autres chercheurs, même si aucun d'entre eux n'est spécialiste de Kierkegaard. J. C. McLelland de McGill semble être fortement influencé par les œuvres les plus connues de Kierkegaard et surtout par l'accent qui y est mis sur le rôle de l'ironie et de l'humour dans la vie religieuse. Ian G. Weeks de l'université McMaster a accompli un travail considérable, comparant la stratégie du Séducteur dans $O u$ bien . . . Ou bien à ce qu'avance Kierkegaard dans Le point de vue. Tout récernment, Barron Brainerd, professeur de linguistique et de statistiques à l'Université de Toronto, a soumis quelques-unes des données de McKinnon sur les pronoms à une analyse statistique plus poussée; il a ainsi voulu utiliser ce matériel pour tenter de démêler les relations complexes entre les écrits nombreux et variés qui seraient l'œuvre d'un seul et même auteur, Kierkegaard. 
Deux événements récents, séparés mais liés, devraient s'avérer décisifs quant à l'évolution des études kierkegaardiennes au Canada. Il nous apparaît donc important de les mentionner ici. Même si j’ai été mêlé à ces deux événements, je vais essayer d'en rendre compte le plus objectivement possible.

Le premier fut l'acquisition par l'université McGill d'environ 1000 volumes de la Collection KierkegaardMalantschuk à l'automne 1979. L'histoire de cette acquisition est compliquée et il ne nous semble pas nécessaire de l'exposer ici en détail. Ce qui est important, c'est que les volumes en question représentent le cœur de la bibliothèque personnelle de M. Malantschuk, qu'une bonne partie de ces volumes furent réunis pendant les dernières années de sa vie et que cette collection comprend une série complète des premières éditions de l'œuvre de Kierkegaard, soit environ 460 livres de la propre bibliothèque de Kierkegaard ou, dans la plupart des cas, des reproductions exactes de ces livres, et environ 500 autres volumes, la plupart datant du siècle dernier, jugés par les responsables de cette acquisition comme étant les ajouts les plus précieux à une collection de recherche de ce genre. Il est peut-être bon de remarquer qu'il s'agit de la collection d'œuvres danoises la plus vaste et la plus riche à pouvoir sortir du Danemark au cours de ce siècle et qu'elle représente déjà la deuxième collection la plus vaste et la plus importante des œuvres de Kierkegaard à quitter le Danemark, la première étant bien sûr la Kierkegaard Library créée par le Professeur et Mme Hong au St. Olaf College de Northfield, Minnesota. Évidemment, c'est un grand honneur pour le Canada et tous les spécialistes des études kierkegaardiennes qui auront à consulter cette collection sont grandement redevables à $M$. Malantschuk pour son sens de la planification, sa prévoyance et sa générosité, ainsi qu'à Mme Grethe Kjær, à M. H. P. Rohde, à Mlle Marianne Scott, à Mlle Allison Cole et à $M$. Hans Möller qui ont tous joué des rôles importants dans l'acquisition de la collection et de son arrivée en bon état à destination. La collection est actuellement gardée dans la salle des livres rares de la bibliothèque MacLennan de McGill, placée sous la responsabilité de Mme Lewis. 
Les personnes que ce genre de collection intéresse constatent rapidement qu'il existe un conflit réel entre le fait de rendre de tels trésors facilement accessibles aux chercheurs et la volonté de préserver ces volumes pour la postérité. Dans une première tentative pour solutionner ce problème, Alastair McKinnon a obtenu de l'Université la permission de faire paraître un volume de microfiches contenant le texte intégral de toutes les premières éditions de la collection et, l'espère-ton, un catalogue complet de toutes les autres œuvres. Si le coût de cette entreprise demeure peu élevé, celle-ci devrait permettre même aux chercheurs individuels d'avoir leurs propres exemplaires des différentes premières éditions, ainsi qu'une information détaillée sur le nouveau matériel de recherche dont dispose maintenant l'université McGill. Il semble fort probable qu'une publication de ce genre jouera un rôle de plus en plus important, étant donné que le prix des voyages augmente, ce qui limite les possibilités de séjour à l'étranger.

Le second événement fut la Conférence Kierkegaard tenue à l'université McGill du 6 au 8 juin 1980. Cette conférence fut conçue comme une suite à la première conférence canadienne sur Kierkegaard qui eut lieu à l'Université de Windsor en 1976 et qui obtint un très grand succès. Cette seconde conférence fut organisée par McKinnon et Khan, assistés de Carignan et Johnson. Elle avait pour but de marquer l'arrivée de la Collection Kierkegaard-Malantschuk, de fournir de l'information sur les diverses ressources et techniques informatiques mises au point à McGill pour l'étude de Kierkegaard et, bien entendu, de fournir une occasion pour les spécialistes de Kierkegaard, en particulier les Canadiens, de se rencontrer et de présenter les uns aux autres les résultats de leurs dernières recherches. D'où le titre : Kierkegaard : Resources and Results. Le sens de ce titre fut bien illustré au cours d'une démonstration, à l'intention des intéressés, de l'utilisation de l'ordinateur pour comparer les première et troisième éditions de Guds Uforanderlighes de Kierkegaard et pour enregistrer toutes les variantes dans chacune de ces éditions. "Que ceux qui ont des yeux voient . . . »

Tout le monde a convenu que cette conférence obtint un franc succès à tous égards. Nous avons compté 68 inscrits, 
dont cinq Danois, un certain nombre d'Américains, un bon nombre de représentants canadiens tant francophones qu'anglophones et des représentants de tous les degrés de compétence, depuis les érudits les plus éminents jusqu’à quelques étudiants de premier cycle. Le premier jour, $M$. Rohde fournit un ensemble d'éléments permettant de comprendre et d'apprécier la Collection Kierkegaard-Malantschuk, alors que cand. teol. Niels Jørgen Cappeløorn présenta les Papirer comme œuvreressource pour la recherche. Au cours de la deuxième journée, le professeur Hong exposa quelques-unes des difficultés que soulève la traduction des textes de Kierkegaard, le professeur Walker traita des problèmes que pose chez Kierkegaard la notion de communauté, le professeur Carignan fit ressortir le rôle synthétisant que joue le concept de l' éternel " dans la pensée de Kierkegaard, et le professeur Nielsen présenta la solution kierkegaardienne à quelques soi-disant problèmes de la pensée religieuse contemporaine. Le dernier jour, le professeur Khan proposa un survol de la "géographie » de la Lidenskab (passion) dans Efterskrift et le professeur McKinnon présenta un modèle des rapports entre les différentes ceuvres dont il fait reposer la "paternité » sur une comparaison des «termes d'une récurrence aberrante » que l'on trouve dans chacune d'elles. Tous ces exposés, auxquels on a ajouté une introduction, ont été réunis, et nous espérons qu'il sera possible de les publier afin de les rendre accessibles à un plus large public. Nous avons tout lieu de croire que cette conférence inaugure une nouvelle ère pour la recherche canadienne sur Kierkegaard. Il ne nous reste plus qu'à remercier la Faculté d'études supérieures et de recherche de l'université McGill, le programme de Formation de chercheurs et d'action concertée du gouvernement du Québec et le Conseil de recherches en sciences humaines du Canada pour leur généreux appui financier.

Étant donné que j’ai été directement mêlé aux recherches sur Kierkegaard, mon jugement risque d'être fort subjectif. J'estime néanmoins que les Canadiens ont déjà apporté une contribution significative à la connaissance de la pensée kierkegaardienne. Jeremy Walker a montré que Kierkegaard a un certain nombre de choses importantes à dire au sein de l'éthique et, ce qui est peut-être encore plus important, qu'il 
rectifie la conception traditionnelle que nous nous faisons de cette discipline. À partir de ses propres études sur Kierkegaard, Ivan Khan est sur le point de restaurer le sentiment et la passion comme thèmes propices à l'investigation et à la réflexion philosophiques. Harry Nielsen a identifié et défait des nœuds subtils de la logique et de la métaphysique kierkegaardiennes, et ce faisant, il a montré, bien que ce soit souvent de manière uniquement implicite, des similitudes et des liens importants avec l'œuvre de Wittgenstein. Ralph Johnson a souligné l'importance capitale de la notion d'existence dans la pensée kierkegaardienne, non pas en tant que concept métaphysique boursouflé et peut-être seulement vide de sens, mais plutôt et simplement comme le trait le plus important de la vie humaine. Maurice Carignan a vu et réaffirmé la profonde intuition de Kierkegaard suivant laquelle les soi-disant problèmes sociaux et politiques des temps modernes sont en réalité d'ordre personnel et, au sens fondamental de ce terme, d'ordre religieux. Cette contribution fut fondamentale et son importance n'est en rien diminuée du fait que plusieurs textes danois sur lesquels elle s'appuyait sont maintenant accessibles en traduction et que deux publications récentes, l'ouvrage de Bruce Kirmmse intitulé Kierkegaard's Politics et le cours célèbre de Malantschuk donné à Londres, ont nettement dissipé tout doute à ce sujet.

McKinnon a fait un certain nombre d'études qui n'ont pas toutes eu le même succès. Ses premiers articles sur l'" irrationalisme " de Kierkegaard distinguent ou supposent cinq sens différents donnés au terme paradoxe dans les écrits de Kierkegaard et, particulièrement, dans ceux de Johannes Climacus; ces divisions nous paraissent maintenant trop grossières et devraient peut-être très retravaillées à la lumière de nouvelles données désormais accessibles. Cependant, la question capitale demeure la même: Kierkegaard est «irrationaliste" si nous mettons l'accent sur la confusion des différents sens qu'il donne au terme paradoxe (voir au terme absurde) et il cesse de l'être lorsque nous distinguons ces sens. Ou bien, pour anticiper, nous dirons qu'il est irrationaliste si nous l'identifions à Johannes Climacus, par exemple, et il cesse de l'être lorsque, tenant compte de ses propres mises en garde, 
nous le distinguons de ce dernier. Dans le même ordre d'idées, McKinnon, dans la première étude informatisée du statut relatif de huit des plus importants pseudonymes de Kierkegaard, adopta malheureusement ces deux conventions anodines séparément, abaissant ainsi excessivement les scores finals pour Philosopbiske Smuler et Afsluttende uvidenskabelig Efterskrift; en effet, l'auteur de ces deux ouvrages ressemble davantage à Kierkegaard que ne le suggèrent les résultats de cette étude. En même temps, il apparaît clairement que ce travail a au moins incité certains autres chercheurs à prendre plus au sérieux les mises en garde de Kierkegaard au sujet du statut des pseudonymes; c'est peut-être là tout ce qu'un chercheur peut souhaiter . . Évidemment, comme McKinnon l'a souligné à l'époque, il est ridicule d'avoir à utiliser l'ordinateur pour convaincre des spécialistes de Kierkegaard que celui-ci savait de quoi il parlait, mais, comme Kierkegaard l'a lui-même bien compris, il y a des situations où l'on est amené à suivre son époque.

Les quatre volumes de The Kierkegaard Indices sont très différents et leurs contributions respectives aux études kierkegaardiennes reflètent naturellement ce fait. Le premier volume consacré à la corrélation entre les pages et les lignes n'était qu'un simple sous-produit de ce projet, mais il a été très apprécié pour la façon dont il facilite la comparaison entre les traductions et dont, en particulier, il permet de mettre en regard les traductions et le texte original. Les deuxième et troisième volumes, Concordance et Index Verborum, ont contribué à réaffirmer l'importance capitale du texte danois et, avec l'excellent Index aux Papirer en trois volumes de Cappelørn, ils ont permis de poser les jalons pour une nouvelle génération de spécialistes de Kierkegaard. Le quatrième volume, Computational Analysis, n'a encore eu que peu d'impact, mais il pourrait bien s'avérer le plus important de tous. Délibérément basé sur le modèle de Kučera et Francis, il exprime une seule opinion (« il apparaît clairement que les différences de style et de contenu entre les divers titres affectent de manière significative la fréquence de l'occurrence même de ces [100] mots danois les plus courants. » p. 857), mais il rapporte littéralement des millions de faits au sujet du statut d'auteur de Kierkegaard. 
Cette analyse part de l'hypothèse suivante: alors que nous pouvons penser en termes de concepts, nous nous exprimons néanmoins au moyen de mots. Nous espérons que l'analyse en question sera toujours accessible lorsque les spécialistes de Kierkegaard auront compris la portée de cette assertion et acquis les techniques statistiques et autres méthodes nécessaires à l'interprétation de ces données.

Depuis plusieurs années, McKinnon utilise certaines techniques de mise à l'échelle statistiques et multidimensionnelles afin de tenter de produire des représentations spatiales éclairées et objectives de la «géographie » de certains concepts-clés de Kierkegaard et, plus récemment, de ses divers ouvrages. Cette méthode est apparue efficace dans le cas de l'étude des concepts, mais il est peut-être encore trop tôt pour juger de sa capacité à identifier et à faire ressortir la structure sous-jacente des ouvrages ; en effet, il semble que, pour en arriver là, il soit nécessaire d'adapter et d'affiner davantage cette méthode. Cependant, on se rend compte déjà que, même à ses débuts, celle-ci peut démontrer que les concepts que l'on dit généralement fondamentaux ne font pas partie de cette structure; du moins pouvons-nous constater que ni le nom de ce concept ni aucun de ses dérivés n'apparaît en fait dans le texte. L'imagination est sans aucun doute un atout important pour un chercheur, mais une connaissance exacte du texte peut parfois lui venir en aide.

J'espère que le lecteur voudra bien me permettre de noter en passant une contribution canadienne minime - mais qui peut s'avérer importante - aux études kierkegaardiennes, contribution qui a déjà été apportée mais qui n'est pas encore tout à fait acceptée. Je veux parler des codes des titres des divers ouvrages utilisés tout au long de ma propre étude, Indices, et basés sur les titres danois même des ouvrages en question. Je plaide en faveur de l'adoption de ces codes pour un certain nombre de raisons fort différentes. Je ne connais aucun autre moyen de ce genre qui soit aussi simple et qui permette à la fois de reconnaître et d'appuyer la primauté du texte danois et de faciliter la communication entre chercheurs travaillant dans des langues différentes; bref, je ne connais aucun autre moyen qui puisse à la fois symboliser et 
promouvoir l'internationalisation des études kierkegaardiennes. La pratique traditionnelle européenne qui consiste à donner le titre du volume et la page, implique qu'il n'y a qu'une seule édition qui soit acceptée, ce qui n'est en fait plus du tout le cas. Des collègues, tant au Canada qu'en Europe, nous ont informé qu'ils utilisent maintenant les codes tels que nous les avons proposés, dans leurs propres écrits et dans leurs recherches, et que cet usage leur paraît tout à fait naturel et utile. Le code du titre peut très bien être suivi du numéro de la page en référence à la fois au texte original danois et à sa traduction, dans l'ordre privilégié ou spécifié par l'auteur, alors qu'un code se référant nettement au titre anglais, par exemple, ne peut pas respecter cet ordre. Pourquoi, par exemple, les Allemands devraient-ils utiliser KT (pour Die Krankeit zum Tode, aussi bien que, notons-le, pour Der Hohepriester - der Zöllner - die Sünderin) et les Français MM. (pour La maladie à la mort) quand nous disposons déjà du Code SD pour Sygdommen til D $\phi$ den? Ou encore pourquoi un chercheur italien devrait-il se rappeler que TWDCF renvoie à Two Discourses at the Communion on Fridays, surtout quand il sait déjà, comme beaucoup d'autres chercheurs, que TAF renvoie à To Taler ved Altergangen om Fredagen? Pourquoi un chercheur, d'où qu'il soit, devrait-il, en rédigeant un article, utiliser un ensemble de simples codes spécifiques fabriqués uniquement à partir de titres anglais, ne comprenant que 15 des 34 livres de l'auteur, et utiliser le même code, CD, pour deux d'entre eux (The Concept of Dread et Discourses at the Communion on Fridays)? Mais ce qui m'amène réellement à prôner l'adoption de ces codes, c'est que je crois que les chercheurs sont des êtres fondamentalement rationnels et que j'ai constaté dans le passé que lorsque mes espoirs ont été déçus, c'est surtout parce que je n’ai pas réussi à les énoncer clairement. Je crois que cette fois je les ai exposés avec suffisamment de clarté.

Un compte rendu sur quelque étude touchant même de loin le Danemark serait incomplet si on ne tenait compte de l'accueil de cette étude non seulement au Danemark mais ailleurs dans le monde. À ce propos, je peux mentionner que le livre de Jeremy Walker a été bien accueilli et que celui de Maurice Carignan a fait l'objet d'une critique favorable. Dans 
les autres cas, nous devons constater que les Canadiens ont à subir les inconvénients qui proviennent de la différence qui existe entre leur approche et celle des Européens. En effet, alors qu'ici on tend à favoriser la concision et la précision (en distinguant, par exemple, les différents sens d'un terme), en Europe on préfere poursuivre des débats sur des œuvres entières, et surtout sur les ouvrages les plus considérables. C'est peut-être pourquoi, sauf exception notable, mes Indices ont été si bien accueillis! De toute façon, l'un de mes nombreux bons souvenirs à cet égard, c'est celui d'une critique par le regretté professeur Sфe dans laquelle celui-ci exprime sa reconnaissance envers ces méthodes de recherche, en même temps que son étonnement qu'elles aient pu provenir du Canada, « un pays qui n'est même pas mentionné dans ses œuvres ». De même, je me réjouis grandement du fait que ces œuvres soient toujours demeurées à l'intérieur des murs de la Søren Kierkegaard Biblioteket de l'Université de Copenhague et que les trois plus grands volumes aient été, il y a longtemps, réunis, copies exactes des originaux, dans une riche et élégante reliure. Peut-être que, à l'extérieur du Canada du moins, le monde est pour ainsi dire trop indulgent à notre égard, à nous Canadiens !

Même si je peux avoir un certain parti-pris, j'estime qu'un brillant avenir se dessine pour les études kierkegaardiennes au Canada et, bien entendu, pour nos études dans le monde entier. Nous avons cet espoir non pas simplement parce que nous possédons la Collection Kierkegaard-Malantschuk, une version assimilable par l'ordinateur de Samlede Varker de Kierkegaard et, à tout le moins, une possibilité d'interroger et d'interpréter ce texte. Aussi importants que puissent être ces éléments, ils ne représentent que les conditions matérielles de la recherche. Ce qui est encore plus important, croyons-nous, c'est un ensemble de conditions dont nous ne sommes pas responsables, mais dont nous sommes néanmoins les heureux bénéficiaires. Nous avons été épargnés des effets désastreux de la tradition Schrempf et nous n'avons nullement éprouvé le besoin de lire et d'interpréter Kierkegaard à travers les yeux de penseurs comme Sartre, Heidegger, Marx, Marcuse, Althusser, Habermas et Adorno qui ont principalement en commun 
d'avoir été quasi aveugles à tout ce que défendait Kierkegaard. Vivant dans deux langues, nous avons appris à ne faire confiance ni à l'une ni à l'autre ; ainsi avons-nous déjà reconnu que c'est au texte danois que nous devons avoir recours en dernière instance. De même, nous profitons de conditions sociales dont l'importance échappe trop souvent aux intellec-

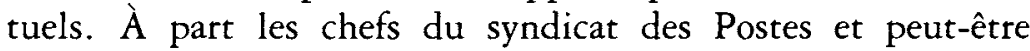
quelques jeunes spécialistes de sciences sociales, presque personne ne croit au Canada que les droits d'un petit groupe aient la priorité absolue sur ceux de la société tout entière. Nous n'avons pas encore succombé aux idéaux confus et contradictoires d'une société égalitaire que Kierkegaard a si clairement démasqués ; notre société n'a pas non plus encore été déchirée et morcelée par cette sorte d'individualisme matérialiste qui est au cœur du marxisme et qui constitue le fondement ouvertement déclaré de son appel aux masses. Certes, les conditions sont loin d'être parfaites, mais nous vivons encore dans une société normale (en termes kierkegaardiens, un état rationnel) au sein de laquelle il est possible à l'individu de se sentir appuyé par la collectivité et, en retour, responsable vis-à-vis de celle-ci, au sein de laquelle il peut considérer le travail et le service comme un privilège et dans laquelle le langage traditionnel du devoir et du sacrifice est au moins partiellement compréhensible. Il n'est pas étonnant alors que la recherche de Carignan ait été l'une des premières à avoir porté sur la pensée sociale de Kierkegaard. Il n'est pas étonnant non plus qu'une récente allocution prononcée par McKinnon, en sa qualité de président de l'Association canadienne de philosophie, ait été intitulée « Kierkegaard as Social Thinker». Mais il ne s'agit là que de quelques exemples particulièrement évidents. Il y a de nombreux autres domaines de recherche pour lesquels nous sommes également bien préparés et il n'en tient qu'à nous de faire un usage réfléchi de richesses dont nous avons hérité. L'expérience montre que si nous menons à bien notre travail, la communauté des chercheurs saura bientôt lui accorder toute la reconnaissance qu'il mérite.

Département de philosophie Université McGill 